\title{
Programa de alimentação do trabalhador e qualidade nutricional das refeições
}

\author{
Worker food program and nutritional quality of meals
}

\author{
Laura Helena Salvettia, Gabriela Possab \\ ${ }^{a}$ Nutricionista. Faculdade Cenecista de Bento Gonçalves, Rio Grande do Sul, Brasil. \\ b Nutricionista. Mestre em Ciências da Saúde, Universidade Federal de Ciências da Saúde de Porto Alegre (UFCSPA). Rio Grande do Sul, Brasil.
}

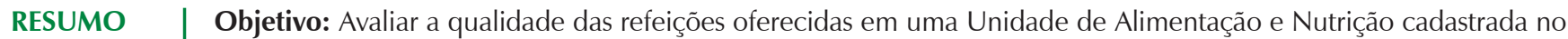
Programa de Alimentação do Trabalhador.

Materiais e Métodos: Do total de cardápios fornecidos, foram selecionados 30\% (71 cardápios) por meio de sorteio sistemático. Os mesmos foram avaliados quanto a sua oferta energética e dos nutrientes carboidratos, proteínas, gorduras totais e saturadas, fibra e sódio. Os valores encontrados foram comparados com os parâmetros estabelecidos pelo Programa de Alimentação do Trabalhador.

Resultados: Observou-se inadequação na oferta energética, a qual ficou em média 60 kcal superior ao limite recomendado, e na contribuição energética da proteína (19\% vs 15\%, este último valor referente à recomendação do Programa de Alimentação do Trabalhador). Em relação à oferta de fibras e sódio, também foram encontrados valores acima da recomendação, no entanto, não foi observado inadequação na oferta de carboidratos, gorduras totais e gorduras saturadas.

Conclusão: No presente estudo, a oferta de macro e micronutrientes mostrou-se inadequada em relação aos parâmetros estabelecidos pelo Programa de Alimentação do Trabalhador.

Palavras-chave: hábitos alimentares; alimentação coletiva; serviços de alimentação.

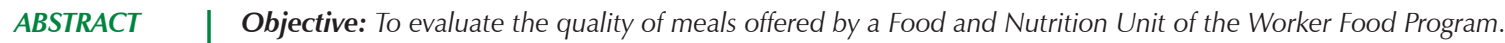

Materials and Methods: From the total of menus provided, 30 percent were selected through a random selection (71 menus). They were assessed regarding their energy supply and their nutrient carbohydrates, proteins, total and saturated fat, fiber, and sodium. Values found were compared to the parameters set by the Worker Food Program.

Results: Inadequacy in the energy supply about $60 \mathrm{kcal}$ above the recommended limit was observed, and in the protein energetic contribution (19\% versus 15\%, being the latter the value recommended by the Worker Food Program). Regarding fiber and sodium supply, values above the recommendation were also found. However, concerning the carbohydrate, total and saturated fat supply, no inadequacy was found.

Conclusion: In the present study, the supply of macro and micronutrients proved inadequate in relation to the parameters established by the Worker Food Program.

Keywords: food habits; collective feeding; food service. 


\section{INTRODUÇÃO}

A população vem adquirindo novos padrões de vida, principalmente no que se relaciona ao trabalho, alimentação e saúde. Dados atuais mostram mudanças nos hábitos e escolhas alimentares ${ }^{1}$, sendo verificado, um aumento no consumo de alimentos com açúcar, gorduras em geral, gorduras saturadas e industrializados e diminuição do consumo frutas e hortaliças, assim como de alimentos básicos como arroz e feijão. Essas mudanças nos hábitos alimentares poderiam explicar as maiores prevalências de excesso de peso visualizadas no País atualmente².

As despesas da população brasileira com alimentação fora do domicílio estão em elevação. Em 2002-2003, 24,1\% das despesas familiares destinavam-se para essas refeições, enquanto em 2008-2009 essa participação aumentou para 31,1\%3․ Em 2014, o mercado de refeições forneceu, em média, 19 milhões de refeições por dia, sendo que as refeições coletivas, produzidas por empresas prestadoras de serviço em Unidades de Alimentação e Nutrição (UAN), representaram 12,2 milhões dessas refeições ${ }^{4}$.

O Programa de Alimentação do Trabalhador (PAT), criado em 1976, teve por objetivo priorizar o atendimento de trabalhadores de baixa renda, ou seja, aqueles que recebem até cinco salários mínimos mensais, além de garantir maior produtividade, melhores condições de saúde, bem-estar e melhor qualidade nutricional das refeições, já que empresas integrantes do PAT devem seguir parâmetros determinados de oferta energética e de nutrientes para as refeições principais (almoço, jantar e ceia) e menores (desjejum e lanche) ${ }^{5}$.

Diante do cenário onde as refeições fora do lar estão cada vez mais frequentes e a saúde da população tem apresentado maior relação com seu consumo alimentar, torna-se importante a avaliação da qualidade nutricional de refeições oferecidas fora do espaço domiciliar. Sendo assim, o objetivo do presente estudo foi verificar a qualidade nutricional de refeições oferecidas a trabalhadores por uma UAN localizada no Sul do Brasil.

\section{MATERIAL E MÉTODOS}

Foi realizado um estudo descritivo que avaliou uma amostra aleatória dos cardápios praticados em uma empresa do ramo metalúrgico, localizada no sul do Brasil, cadastrada no Programa de Alimentação do Trabalhador (PAT). No momento do estudo, a empresa fornecia a refeição almoço para aproximadamente 150 trabalhadores das áreas operacionais e administrativas, sendo em sua maioria homens (76\%).

O cardápio é elaborado por uma nutricionista a partir de itens definidos em contrato corporativo entre a UAN terceirizada e a empresa. O cardápio é composto por dois tipos de saladas, dois tipos de sobremesa (doce ou fruta), arroz, feijão, duas guarnições, sendo uma delas a sopa, pão, suco, dois tipos de carnes e preparações utilizando ovos. Referente à sobremesa e às carnes e ovos, os trabalhadores precisam fazer uma escolha entre as opções: fruta ou doce, no caso da sobremesa, e dois tipos de carne ou um tipo de carne e preparações à base de ovos, no caso das carnes e ovos.

Para o presente estudo, foram avaliados cardápios obtidos no período de 01/01/2014 a 31/12/2014, resultando em 237 dias/cardápios possíveis de serem analisados, já que a UAN não produz refeições aos sábados, domingos, feriados e período de férias da empresa. Deste total de dias, realizou-se a seleção, por meio de sorteio sistemático com início casual e intervalo igual a 2, de 30\% dos cardápios do almoço, resultando em uma amostra de 71 cardápios $^{6}$. A avaliação dos cardápios teve como objetivo estimar o valor energético ofertado e o perfil dos nutrientes: carboidratos, proteínas, gorduras totais, gorduras saturadas, fibra e sódio, utilizando como referência os parâmetros expostos na Tabela 1.

Tabela 1. Parâmetros nutricionais estabelecidos pelo programa de alimentação do trabalhador (PAT).

\begin{tabular}{lcc}
\hline \multicolumn{1}{c}{ Nutriente } & $\begin{array}{c}\text { Recomendação } \\
\text { diária }\end{array}$ & $\begin{array}{c}\text { Recomendação } \\
\text { refeição principal }\end{array}$ \\
\hline Valor Energético (kcal)* & 2000 & $600-800$ \\
Carboidratos (\%) & $55-75$ & 60 \\
Proteína (\%) & 15 & 15 \\
Gorduras Totais (\%) & $15-30$ & 25 \\
Gorduras Saturadas (\%) & $<10$ & $0-10$ \\
Fibra (g) & $>25$ & $7-10$ \\
Sódio (mg) & $\leq 2400$ & $720-960$ \\
\hline
\end{tabular}

Fonte: Ministério do Trabalho e Emprego (MTE), $2006^{5}$.

As fichas técnicas foram elaboradas pela responsável pela pesquisa através das receitas já existentes na UAN. Primeiramente, as receitas foram selecionadas, após os rendimentos e as porções ofertadas aos comensais foram obtidas através de pesagem e relato da equipe. Os cálculos foram realizados por meio do programa Dietwin ${ }^{\circledR}$-Professional Versão 2008, utilizando como referência os alimentos da Tabela de Composição de Alimentos ${ }^{7}$. No entanto, quando o alimento não estava citado nessa tabela, foram utilizados dados encontrados na Tabela de Composição Nutricional dos Alimentos Consumidos no Brasil ${ }^{8}$ e, complementarmente, buscou-se o rótulo de alguns alimentos presentes nos cardápios. Ressalta-se, porém, que os temperos adicionados às saladas (sal, óleo de soja, vinagre, molho shoyu, molho inglês e molho de pimenta) não foram adicionados ao cálculo dos cardápios, pois não existia um registro do consumo diário desses produtos. No cálculo das proteínas e das 
sobremesas foi utilizada uma média ponderal, considerando uma estimativa de proporção de escolha de cada um dos itens disponíveis no cardápio baseada no relato da equipe de UAN.

A estimativa da quantidade energética e de nutrientes oferecida e a contribuição energética nos macronutrientes foi apresentada como média, desvio padrão, mediana e percentis 25 e 75 dos 71 cardápios. Por fim, foram comparadas as médias, no caso de distribuição simétrica, ou medianas, no caso de distribuição assimétrica, dos valores de oferta energética e dos nutrientes (proteína, carboidrato, gorduras totais, gorduras saturadas, fibras e sódio) com os parâmetros nutricionais estabelecidos pelo PAT. As análises descritivas foram efetuadas no programa Statistical Package for Social Sciences (SPSS) versão 21.0.

O presente estudo não foi submetido à análise do comitê de ética da instituição, já que não envolveu indivíduos. No entanto, as empresas envolvidas autorizaram a coleta de dados para a pesquisa.

\section{RESULTADOS}

A análise realizada demonstrou inadequação no valor energético médio dos cardápios calculados. A média encontrada para oferta energética foi de $861,4 \pm 141,5 \mathrm{kcal}$, excedente ao limite de 30 a $40 \%$ do valor energético total (VET) diário de $2000 \mathrm{kcal}$ recomendado pelo PAT, no entanto, foram constatados que $25 \%$ dos cardápios apresentaram valor energético entre $604,0 \mathrm{kcal}$ e $762,2 \mathrm{kcal}$, valores adequados à recomendação (Tabela 2).

$\mathrm{Na}$ avaliação da contribuição energética dos macronutrientes, observaram-se valores discrepantes ao recomendado pelo PAT, sendo que, para os carboidratos e gorduras totais a mediana de contribuição ficou abaixo da recomendação (56,2\% e $23,9 \%$, respectivamente) e, para as proteínas, acima da recomendação (19\%). Exceção foi observada para as gorduras saturadas que apresentaram contribuição energética adequada (6,7\%). No entanto, na comparação entre os valores das medianas em $\mathrm{g} / \mathrm{dia}$ observados com os valores sugeridos pelo PAT, observou-se que para os carboidratos e gorduras totais os valores apresentaram-se de acordo com a recomendação $(117,3 \mathrm{~g}$ e $20,7 \mathrm{~g}$, respectivamente), já para as proteínas, o resultado permaneceu elevado $(40,8 \mathrm{~g})$ (Tabela 2 e 3 ).

Referente às fibras, foi possível visualizar que a média de oferta da mesma foi de 12,8 $\pm 2,2 \mathrm{~g}$ superando o valor máximo de $10 \mathrm{~g}$ descrito pelo PAT. No tocante ao sódio, observaram-

Tabela 2. Estimativa da quantidade energética e de nutrientes oferecidas pela empresa estudada.

\begin{tabular}{|c|c|c|c|c|c|c|}
\hline Nutrientes & Média \pm DP & Mínimo & P25 & Mediana & P75 & Máximo \\
\hline Energia (kcal) & $861,46 \pm 141,5$ & 604,0 & 762,2 & 848,2 & 923,2 & 1266,1 \\
\hline Carboidratos (g) & $121,65 \pm 22,1$ & 86,3 & 105,2 & 117,3 & 131,4 & 188,3 \\
\hline$(\%)^{*}$ & $56,59 \pm 5,2$ & 39,7 & 54,3 & 56,2 & 59,5 & 69,8 \\
\hline Proteínas (g) & $42,52 \pm 9,4$ & 26,4 & 38,2 & 40,8 & 46,4 & 100,0 \\
\hline$(\%)^{*}$ & $19,93 \pm 3,6$ & 13,0 & 17,5 & 19,0 & 21,8 & 32,0 \\
\hline Gorduras Totais (g) & $22,74 \pm 7,7$ & 10,5 & 17,3 & 20,7 & 28,5 & 48,1 \\
\hline$(\%)^{*}$ & $23,47 \pm 5,5$ & 13,2 & 18,8 & 23,9 & 27,6 & 37,3 \\
\hline Gorduras Saturadas (g) & $6,38 \pm 2,6$ & 1,6 & 4,7 & 5,9 & 7,4 & 15,3 \\
\hline$(\%)^{*}$ & $6,56 \pm 2,1$ & 2,0 & 4,7 & 6,7 & 7,7 & 11,9 \\
\hline Fibras (g) & $12,84 \pm 2,2$ & 9,2 & 11,4 & 12,5 & 14,1 & 18,7 \\
\hline Sódio (mg) & $2114,74 \pm 524,9$ & 597,0 & 1772,5 & 2141,9 & 2367,7 & 3731,8 \\
\hline
\end{tabular}

* \% de contribuição energética. DP: desvio padrão.

Tabela 3. Valores médios encontrados e recomendação máxima e mínima preconizada pelo programa de alimentação do trabalhador (PAT).

\begin{tabular}{|c|c|c|c|c|c|c|}
\hline \multirow{2}{*}{ Nutriente } & \multicolumn{2}{|c|}{ Média/mediana dos 71 cardápios } & \multicolumn{2}{|c|}{ Valores mínimos PAT } & \multicolumn{2}{|c|}{ Valores máximos PAT } \\
\hline & $\mathrm{g} / \mathrm{mg}$ & $\% *$ & $\mathrm{~g} / \mathrm{mg}$ & $\% *$ & $\mathrm{~g} / \mathrm{mg}$ & $\% *$ \\
\hline Valor Energético (kcal) & 861,5 & - & 600,0 & - & 800,0 & - \\
\hline Carboidrato $(g)^{\S}$ & 117,3 & 56,2 & 90,0 & 60,0 & 120,0 & 60,0 \\
\hline Proteína $(g)^{\S}$ & 40,8 & 19,0 & 22,5 & 15,5 & 30,0 & 15,0 \\
\hline Gorduras Totais $(\mathrm{g})^{\S}$ & 20,7 & 23,9 & 16,6 & 25,0 & 22,2 & 25,0 \\
\hline Gorduras Saturadas $(\mathrm{g})^{\varsigma}$ & 5,9 & 6,7 & 0,0 & 0,0 & 8,8 & 10,0 \\
\hline Fibras (g) & 12,8 & - & 7,0 & - & 10,0 & - \\
\hline Sódio (mg) & 2114,7 & - & 720,0 & - & 960,0 & - \\
\hline
\end{tabular}

*\% de contribuição energética. ${ }^{\S}$ mediana. 
se valores superiores ao recomendado pelo PAT já a partir do percentil 25 (1772,5 mg). O valor médio encontrado foi 2,2 vezes superior à recomendação $(2114,7 \pm 524,9 \mathrm{mg})$, enquanto o percentil 75 foi quase quatro vezes superior (3731,8 mg) (Tabela 2 e 3).

\section{DISCUSSÃO}

O presente estudo avaliou refeições servidas por uma empresa cadastrada no Programa de Alimentação do Trabalhador e observou inadequações nutricionais em alguns aspectos como a oferta energética e dos nutrientes proteínas, fibras e sódio.

Outros estudos também avaliaram a qualidade das refeições oferecidas em UAN e observaram que a oferta de nutrientes apresenta desvios, tornando as refeições oferecidas muitas vezes inadequadas nutricionalmente quando comparadas às recomendações do PAT. Dentre os desvios, observa-se excesso na oferta calórica e proteica, o que corrobora os resultados do presente estudo. Porém, diferentemente deste estudo, alguns estudos encontraram oferta excessiva de gorduras totais e saturadas $6,9,10$.

O Guia Alimentar da População Brasileira sugere uma alimentação adequada e equilibrada como forma de prevenção de doenças crônicas não transmissíveis ${ }^{11}$. Em um estudo realizado em uma população de trabalhadores foi observado que um terço deles apresentava obesidade. Isso demonstra a importância de uma refeição adequada no que diz respeito à oferta energética ${ }^{12}$.

Em relação à análise proteica, ressalta-se que o presente estudo utilizou uma estimativa de proporção de consumo dos diferentes pratos ofertados, já que havia uma possibilidade de escolha do comensal às proteínas (carnes e ovos) ofertadas. Esta estimativa pode ter superestimado o valor de oferta proteica encontrado. Além disso, a avaliação proteica não teve como objetivo a análise da qualidade desse nutriente, no que se refere à digestibilidade e composição de aminoácidos.

O presente estudo encontrou inadequações na oferta de fibras e sódio, o que corrobora os resultados encontrados por outros estudos, sendo a oferta de ambos nutrientes superior às recomendações ${ }^{13,14}$. O consumo de fibras apresenta efeitos positivos para a saúde tal como a redução nos níveis séricos de colesterol, melhora na glicemia em pacientes com diabetes, redução de peso corporal, redução de processos inflamatórios de baixo grau, dentre outros ${ }^{15}$. A recomendação atual para consumo de fibras segundo o Ministério da Saúde é de $25 \mathrm{~g} / \mathrm{dia}^{1}$, o que condiz com a recomendação do PAT. Os dados encontrados no presente estudo indicaram uma oferta superior de fibras. Assim, considerando a alimentação da população brasileira, que tem apresentado baixo consumo de frutas e verduras e, consequentemente, de fibras ${ }^{16}$, podemos considerar que os valores elevados encontrados no presente estudo não são um problema e, sim, um aspecto positivo.

No presente estudo, foi observado uma oferta média de sódio que superou a recomendação total de sódio diária para a população brasileira ${ }^{1}$, o que é preocupante se considerarmos as evidências que apontam a relação do seu consumo com doenças crônicas como hipertensão, doenças cardiovasculares $^{17,18}$, acidente vascular cerebral e doenças renais ${ }^{19}$. Estudo recente avaliou a percepção do consumo de sal pela população brasileira e demonstrou que, aproximadamente, um em cada seis indivíduos classifica seu consumo de sal como elevado ${ }^{20}$. A estimativa de consumo de sódio realizada através da Pesquisa de Orçamentos Familiares (POF 2008-2009) constatou um consumo médio 4,7g de sódio em uma dieta de $2000 \mathrm{kcal}^{21}$, sendo esse valor superior ao limite recomendado pelo Ministério da Saúde que é de $2 \mathrm{~g} / \mathrm{dia}^{22}$.

O Guia de Boas Práticas Nutricionais para Restaurantes Coletivos orienta que a quantidade de sódio deve ser reduzida gradualmente nas refeições fornecidas por UAN. Sendo assim, é sugerida a substituição do sal comum pelo sal com ervas secas, o qual utiliza uma parte de sal para três partes de ervas, ou a produção de caldos utilizando como base legumes e carnes afim de evitar o consumo de caldos e temperos industrializados ${ }^{23}$.

O presente estudo apresentou algumas limitações. Primeiramente, não se aplicou inquéritos alimentares para avaliar o consumo dos comensais e o resto ingestão dos alimentos oferecidos. Além disto, os temperos utilizados nas saladas, no qual estão inclusos sal e óleo, podem ter subestimado os valores encontrados de sódio e de lipídeos.

Considerando a metodologia utilizada, é possível concluir que o PAT vem sendo falho em UAN. Sabe-se que esses locais deveriam levar em consideração o público que estão atendendo e que uma alimentação desequilibrada, com excesso na oferta energética de alguns nutrientes e déficit de oferta de outros, pode gerar graves consequências à saúde do trabalhador.

\section{REFERÊNCIAS}

1. Ministério da Saúde (BR). Secretaria de Atenção à Saúde. Guia alimentar para a população brasileira: promovendo a alimentação saudável. Brasília: Ministério da Saúde; 2008.

2. Levy-Costa RB, Sichieri R, Pontes NS, Monteiro CA. Disponibilidade domiciliar de alimentos no Brasil: distribuição e evolução (19742003). Rev Saúde Pub. 2005;39(4):1-10. https://doi.org/10.1590/ s0034-89102005000400003

3. Instituto Brasileiro de Geografia e Estatística. Pesquisa de orçamentos familiares 2008-2009: aquisição alimentar domiciliar per capita: Brasil e grandes regiões. Rio de Janeiro: IBGE; 2010. 
4. Associação Brasileira das Empresas de Refeições Coletivas. Mercado Real de Refeições [Internet]. São Paulo: ABERC; 2015 [cited 2015 Set 7]. Available from: http://www.aberc.com.br

5. Ministério do Trabalho e do Emprego (BR). Programa de Alimentação do Trabalhador - PAT [internet]. [cited 2016 Maio 15]. Available from: http://acesso.mte.gov.br/pat/programa-de-alimentacao-dotrabalhador-pat.htm

6. Gorgulho BM, Lipi M, Marchioni DML. Qualidade nutricional das refeições servidas em uma unidade de alimentação e nutrição de uma indústria da região metropolitana de São Paulo. Rev Nutr. 2011;24(3):463-72. https://doi.org/10.1590/S141552732011000300009

7. Núcleo de Estudos e Pesquisas em Alimentação - NEPA, Universidade Estadual de Campinas. Tabela brasileira de composição de alimentos - TACO. 4ae ed. Campinas: NEPA-UNICAMP; 2011.

8. Instituto Brasileiro de Geografia e Estatística. Pesquisa de orçamentos familiares 2008-2009: Tabelas de composição nutricional dos alimentos consumidos no Brasil. Rio de Janeiro: IBGE; 2011.

9. Carneiro NS, Moura CMA, Souza SCC. Avaliação do almoço servido em uma unidade de alimentação e nutrição, segundo os critérios do Programa de Alimentação do Trabalhador. Braz J Food Nutr. 2013;24(3):361-5.

10. Sousa FA, Silva RCO, Fernandes CE. Avaliação nutricional de cardápios em unidades de alimentação e nutrição: Adequação ao Programa de Alimentação do Trabalhador. Veredas-Favip Rev Eletr de Cienc. 2009;2:43-50.

11. Ministério da Saúde (BR). Secretaria de Atenção à Saúde. Departamento de Atenção Básica. Guia alimentar para a população brasileira. 2aㅡ ed. Brasília: Ministério da Saúde; 2014.

12. Silveira PM, Silva KS, Silva JA, Oliveira ESA, Barros MVG, Nahas MV. Overweight in Brazilian industry workers: Prevalence and association with demographic and socioeconomic factors and soft drink intake. Rev Nutr. 2014;27(4):435-45. https://doi.org/10.1590/141552732014000400005

13. Lagemann MD, Fassina P. Avaliação da composição nutricional de refeições oferecidas em uma unidade de alimentação e nutrição. Cad Pedagog. 2015;12(1):36-45.
14. Rocha MP, Matias ACG, Spinelli MGN, Abreu ES. Adequação dos cardápios de uma unidade de alimentação em relação ao programa de alimentação do trabalhador. Rev Univap. 2014;20(35):112-8. https://doi.org/10.18066/revunivap.v20i35.211

15. Bernaud FSR, Rodrigues TC. Fibra alimentar: ingestão adequada e efeitos sobre a saúde do metabolismo. Arq Bras Endocrinol. 2013;57(6):397-405. https://doi.org/10.1590/S0004-27302013 000600001

16. Instituto Brasileiro de Geografia e Estatística. Pesquisa de orçamentos familiares 2008-2009: análise do consumo alimentar pessoal no Brasil. Rio de Janeiro: IBGE; 2011.

17. Dickinson BD, Havas S. Reducing the population burden of cardiovascular disease by reducing sodium intake: a report of the Council on Science and Public Health. Arch Intern Med. 2007;167(14):1460-8. https://doi.org/10.1001/archinte.167.14. 1460

18. Zhao D, Qi Y, Zheng Z, Wang Y, Zhang XY, Li HJ, Liu HH, Zhang $\mathrm{XT}, \mathrm{Du}$ J, Liu J. Dietary factors associated with hypertension. Nat Rev Cardiol. 2011;8:456-65. https://doi.org/10.1038/nrcardio. 2011.75

19. He FJ, MacGregor GA. Reducing population salt intake worldwide: from evidence to implementation. Prog Cardiovasc Dis. 2010;52(5):363-82. https://doi.org/10.1016/j.pcad.2009.12.006

20. Oliveira MM, Malta DC, Santos MAS, Oliveira TP, Nilson EAF, Claro RM. Consumo elevado de sal autorreferido em adultos: dados da Pesquisa Nacional de Saúde, 2013. Epidemiol Serv Saúde. 2015;24(2):249-56. https://doi.org/10.5123/S167949742015000200007

21. Sarno F, Claro RM, Levy RB, Bandoni DH, Monteiro CA. Estimativa de consumo de sódio pela população brasileira, 2008-2009. Rev Saúde Publ. 2009; 47(3):571-8. https://doi.org/10.1590/S00348910.2013047004418

22. World Health Organization. Guideline: Sodium intake for adults and children. Geneva: WHO; 2012.

23. Agência Nacional de Vigilância Sanitária (BR). Guia de Boas Práticas Nutricionais: restaurantes coletivos. Brasília: Ministério da Saúde; 2014. 